\title{
ESTUDO APLICADO SOBRE O USO DE JOGOS EDUCATIVOS NO ENSAIO NA TABUADA
}

\author{
Daniela Emilena Santiago ${ }^{111}$
}

\begin{abstract}
RESUMO
O presente artigo teve como objetivo apresentar e analisar as possibilidades de integração entre as práticas pedagógicas no quinto ano do Ensino Fundamental e o uso das Tecnologias da Informação e Comunicação (TIC) através de jogos educacionais no processo de ensino da tabuada. Trata-se de um estudo de campo com abordagem quantitativa, realizado com a intenção de apontar possibilidades do uso de tecnologias educacionais que ofereçam suporte didático pedagógico aos professores da rede pública municipal da Secretaria da Educação da cidade de Palmital. O jogo educativo foi escolhido dentre uma grande variedade de opções oferecidas na internet por se tratar de um jogo com acesso gratuito de fácil manuseio, dinâmico e colorido que apresenta a tabuada e desperta no aluno o interesse em resolver problemas brincando e aprendendo a utilizar a tabuada, dando mais segurança no desenvolvimento das atividades propostas. No estudo proposto foram aplicadas avaliações antes e depois da utilização do software. Os dados foram analisados de forma comparativa e estatística concluindo que o software proporcionou resultado satisfatório em alunos no sentido de construção do conhecimento sobre a tabuada.
\end{abstract}

Palavras-chave: Jogos Educativos, Matemática, Tabuada.

\begin{abstract}
This article aims to present and analyze the possibilities of integration between pedagogical practices in the fifth year of elementary school and the use of Information and Communication Technologies (ICT) through educational games in the process of teaching multiplication tables. This is a field study with a quantitative approach, with the intention of pointing out possibilities of the use of educational technologies that offer pedagogical didactic support to the teachers of the municipal public school of the Secretary of Education of the city of Palmital. The educational game was chosen from a wide variety of options offered on the Internet because it is a free game with easy access, dynamic and colorful that presents the multiplication table and arouses the student's interest in solving problems by playing and learning to use the multiplication table., giving more security in the development of the proposed activities. In the proposed study, evaluations were applied before and after the use of the software. The data were analyzed comparatively and statistically concluding that the software provided satisfactory results in students in the construction of knowledge about the multiplication table.
\end{abstract}

Keywords: Educational Games, Math, Multiplication Table.

\footnotetext{
${ }^{111}$ Possui graduação em Serviço Social pela Universidade Estadual de Londrina, especialização em Violência Doméstica contra crianças e adolescentes pela Universidade de São Paulo, Mestrado em Psicologia e em História pela Universidade Estadual Paulista Julio de Mesquita Filho e atualmente cursa Doutorado em História pela Universidade Estadual Paulista Julio de Mesquita. Docente dos cursos de Pedagogia e Psicologia da UNIP, campus Assis.
} 


\section{INTRODUÇÃO}

O mundo contemporâneo está marcado por avanços significativos na comunicação, na área tecnológica, transformações científicas, de forma a afetar diretamente o cotidiano. Esse fato acentua a necessidade de incorporar novos métodos pedagógicos, mais atrativos para crianças e adolescentes. A prática pedagógica enfrenta o desafio de buscar novas estratégias de ensino para que o educando se aproprie de maneira satisfatória do conhecimento adquirido com as novas tecnologias (KOCH, 2013).

As tendências na Educação, Tecnologia da Informação e Comunicação (TIC) tem recebido destaque dado às contribuições que essas ferramentas podem oferecer aos processos de ensino e aprendizagem por meio de uma grande variedade de softwares e dispositivos. A utilização de tecnologias digitais tem sido algo presente em todas as áreas da sociedade contemporânea, como afirma Rankine (1987) e vem sendo utilizadas oportunizando o aprendizado de uma forma mais contextualizada com o cotidiano.

\footnotetext{
Testemunhar o desenvolvimento de uma capacidade até agora não imaginada de ampliar o intelecto humano. O homem tem uma capacidade singular de armazenar informação e utilizá-las para o seu progresso e bem estar. A tecnologia da sociedade de informação amplia esta capacidade humana, bem além de qualquer nível julgado possível a um quarto de século, colocando conhecimentos à disposição dos que necessitam, quando necessitam e onde quer que estejam. (RANKINE, 1987, p.292).
}

O processo de ensino aprendizagem da matemática trouxe vinculado consigo o ensino da tabuada, que é um instrumento didático-pedagógico imprescindível para a realização de cálculos matemáticos básicos de multiplicação e divisão. O contexto de ensino da matemática, assim como da tabuada nas escolas de ensino fundamental, vem sendo modificado com o passar do tempo, assim como as abordagens de ensino como, por exemplo a transição do Tradicionalismo para o Construtivismo (PEREIRA, 2016).

Desde a implantação do ensino utilizando o método tradicional até os tempos atuais, existiram alterações significativas no modo de ensinar a utilizar a tabuada, considerando o seu formato de apresentação e metodologia para o aprendizado das operações aritméticas (DANI e GUZZO, 2013).

O desafio é acompanhar os alunos do século XXI, imersos em uma acelerada propagação de informações e apropriação do conhecimento e em decorrência disso, os processos educacionais sofrem contínuas influências e mudanças, demandando métodos de ensino e aprendizagem cada vez mais atualizados (PEREIRA, 2017).

O que se entende por escola tradicional remete à forma de organização de grupo escolar, com salas de aula separadas de acordo com o nível de conhecimento do aluno ("adiantados" e "atrasados"), e 
cuja transferência do conteúdo se dá de modo mecânico. Segundo Valente e Pinheiro (2013), não havia muito ou quase nenhum material didático que pudesse orientar a prática do professor com métodos diferenciados:

[...] numa ambiência onde o material didático era praticamente inexistente, sedimentase a memorização da Tábua de Pitágoras, pois através dela será possível obter e memorizar os resultados das multiplicações de números naturais. Trata-se de um dos raros dispositivos didáticos a ganhar lugar no ensino da Aritmética, do Cálculo, em tempos de inexistência de livros e materiais didáticos para os alunos. (VALENTE E PINHEIRO, 2013, p.6).

O surgimento da pedagogia moderna, amparada pela Base Nacional Comum Curricular (BNCC) de 2017, propõe que o ensino de matemática seja abordado de forma mais reflexiva e menos decorada. O professor deve desenvolver no aluno a habilidade de raciocínio lógico, e incentivar o uso inteligente de seus recursos, propondo soluções para os problemas do dia a dia, tanto na escola, quanto fora dela, tendo em vista que a resolução de problemas é um processo para se ter estratégia, e com isso promover o processo de matematização. A multiplicação significa adicionar quantidades iguais, e mediante essa concepção é que se justifica a representação com materiais manipuláveis que vêm consolidar a ideia multiplicativa da tabuada. Dessa maneira, há a possibilidade do aluno pensar em outras formas de resolução e se sinta mais motivado para novas descobertas (PEREIRA, 2016).

Autores, como Souza (2018), defende a necessidade de memorização da tabuada, pois é de muita importância para que o aluno domine e aprenda algumas técnicas de cálculo. Assim, explorando outros conteúdos matemáticos (frações, geometria, múltiplos, divisores, entre outros) a multiplicação aparecerá com muita frequência. Com isso, se caso o aluno não tiver memorizado, sempre perderá tempo e desviará a sua atenção construindo a tabuada, ou contando nos dedos. Para Santos (2014), ao ensinar matemática é necessário desenvolver no aluno o raciocínio, a criatividade e sua capacidade de resolver problemas.

A tabuada é cobrada pelos professores desde os quatro primeiros anos das séries iniciais, e mesmo assim chegam no quinto ano com dificuldades nas resoluções de problemas supostamente causadas pela falta de memorização da mesma (OLIVEIRA, 2008).

O processo educacional tem passado por transformações significativas. Desde o século XVII quando Comênio, pesquisador tcheco, considerado o pai da Pedagogia Moderna começou a sistematizar a pedagogia e a didática na educação escolar, variadas pesquisadas têm sido desenvolvidas para tornar o processo ensino aprendizagem mais satisfatório tanto para os alunos quanto para os professores. Neste contexto, a tecnologia, aliada a educação, desempenha um papel crucial nas conexões que acontecem entre a escola e a sociedade moderna imersa em inovações. Por isso, se faz tão necessária a utilização das tecnologias de informação e comunicação (TICs) s nos espaços escolares (DOWBOR, 2001, p.11). 
Assim, esse trabalho visa destacar alguns aspectos relacionados a necessidade de incorporar novas tecnologias no processo pedagógico, com o intuito de colaborar com o processo de ensino-aprendizagem da tabuada.

\section{A TECNOLOGIA NO ENSINO DA TABUADA}

O uso de atividades lúdicas desperta nos alunos a capacidade de aprendizagem e favorecerem o raciocínio lógico. Segundo Starepravo (2010) utilizar jogos nas aulas de matemática é uma possibilidade

112 de diminuir bloqueios apresentados por muitos alunos que temem a matemática e se sentem incapacitados para aprendê-la.

Dani e Guzzo (2013) reforçam a importância de o professor criar condições para que o aluno perceba que a matemática está presente no seu cotidiano, citando fatos, momentos significativos para aguçar o interesse pela matéria. Uma boa maneira de colocar em prática essa ação é trabalhar com recursos lúdicos, partir de material manipulável e dos jogos eletrônicos, por exemplo, que estão presentes nas teorias e documentos oficiais que norteiam a prática escolar. O uso da tecnologia com finalidade pedagógica visa principalmente à integração dos alunos e professores, buscando compreender e interpretar fenômenos socioculturais bem como o envolvimento em atividades sociais relevantes (FREIRE e VALENTE, 2001).

De acordo com Kishimoto (2012), em situações de jogos as crianças desenvolvem uma interação umas com as outras, aprendendo a trabalhar em grupos, cooperando, respeitando regras, assumindo responsabilidades e aceitando penalidades.

Segundo Freire e Valente (2001), o uso da tecnologia com finalidade pedagógica visa principalmente à integração dos alunos e professores, buscando compreender e interpretar fenômenos socioculturais bem como o envolvimento em atividades sociais relevantes.

Dentre as tendências em Educação, as TICs têm recebido destaque dado às contribuições que essas ferramentas podem oferecer ao ensino da matemática por meio de uma grande variedade de softwares educacionais, dispositivos como computadores, tablets, smartphones, lousas digitais dentre outros, tendo o professor como um mediador e auxiliando neste processo. O uso destas ferramentas permite acessar os mais variados conteúdos didáticos, podendo promover a interdisciplinaridade de uma forma intuitiva, atraente e lúdica (VESCE, 2019).

Segundo Grubel e Bez (2006), a criança que usa jogos tem mais facilidade para enfrentar problemas na vida, pois desenvolve a habilidade de entender o significado de ganhar ou perder. Assim, os alunos desenvolvem capacidades, habilidades, encontar conhecimentos e atitudes, além de auxiliar na construção da sua autoconfiança. Abaixo encontram-se alguns autores que utilizaram os jogos como método para aprendizagem. 
Ventorini (2014), utilizou jogos para o aprendizado com a utilização dos jogos Tabuada do Dino (escola games) e o Labirinto da tabuada, analisando os resultados pode-se perceber um aumento da aprendizagem dos cálculos matemáticos em cem por cento dos alunos, de forma significativa, foi realizado um comparativo entre os testes antes e depois da utilização dos jogos, alguns alunos melhoraram suas notas de 50 para 90, de 60 para 90, assim como alguns não melhoraram tanto assim mas já houve uma evolução significativa pois pode-se analisar também alguns aumentos de 30 para 50, de 20 para 35, sendo esses alunos, aqueles que apresentam grandes dificuldades de aprendizagens.

Fagundes e Falkembach (2013) aplicaram o jogo online LEMONADE nos alunos do quarto ano

113 fundamental, chegando à conclusão que o trabalho só foi valido no momento em que os alunos começaram a se comunicar avaliando os resultados uns dos outros e se ajudando para que tivessem um bom desempenho, demonstrando alegria, motivação e interesse nos cálculos.

Lima et al. (2015), utilizaram o software TuxMath livre, com o objetivo de sanar as dificuldades dos alunos nas quatro operações, contribuir na compreensão das diferentes estratégias ligadas à resolução de situações problemas, além de uma forma de trabalho divertida e prazerosa. Outro software usado foi o Gcompris, usado para trabalhar diversas habilidades cognitivas, com uma interface bem atrativa, ideal para trabalhar com estudantes na educação infantil e ensino fundamental. O resultado deste trabalho é que as notas dos alunos e o interesse melhoraram significativamente, o que surpreenderam os professores. Além disso, observou-se que a apreensão de conteúdos relacionados ao conteúdo matemático, em especial com relação a tabuada melhorou significativamente. Para maiores detalhes apresentamos a pesquisa na sequência.

\section{A APLICAÇÃO DO JOGO EDUCATIVO DIGITAL NA CONSTRUÇÃO DO CONHECIMENTO DA TABUADA.}

Foram realizadas atividades com o jogo educativo digital chamado "Brincando com Ariê", em uma escola municipal da cidade de Palmital, com a participação de 20 alunos do $5^{\circ}$ ano do Ensino Fundamental do turno matutino.

Primeiro, foi realizada uma aula expositiva sobre a tabuada de forma convencional utilizando a lousa e materiais concretos. Após o ensino da tabuada, usando uma abordagem tradicional, os alunos foram avaliados por meio da resolução de 20 exercícios de multiplicação.

Os alunos foram levados ao laboratório da escola para apresentação do software. Os mesmos se sentaram em duplas e foi feita uma explicação de como deveriam utilizar os jogos. Cada aluno desenvolveu as atividades propostas individualmente. As aulas no laboratório de informática foram realizadas durante uma hora semanal, no período de quatro semanas. O acesso ao jogo foi realizado através do site "http://www.brincandocomarie.com.br/arie-tabuada/" (Figuras 1 e 2).

Figura 1- Tela inicial do jogo Brincando com Ariê.

Revista Lumen, v. 5, $\mathrm{n}^{\circ}$ 9, Jan./Jun. - 2020 - ISSN: 2447-8717 


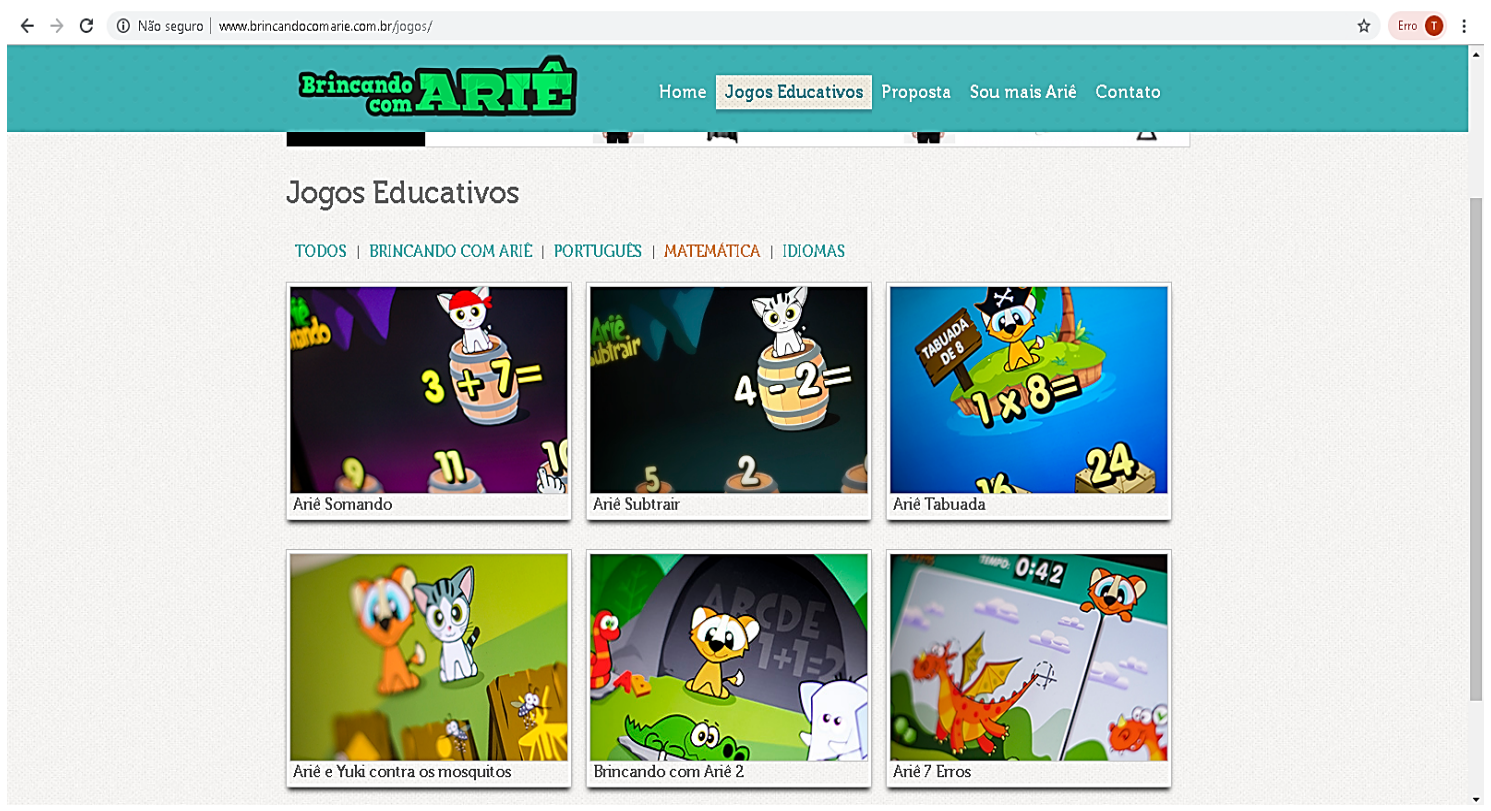

Fonte: Brincando com Ariê, 2019.

Figura 2 - Explorando a tabuada do dois.

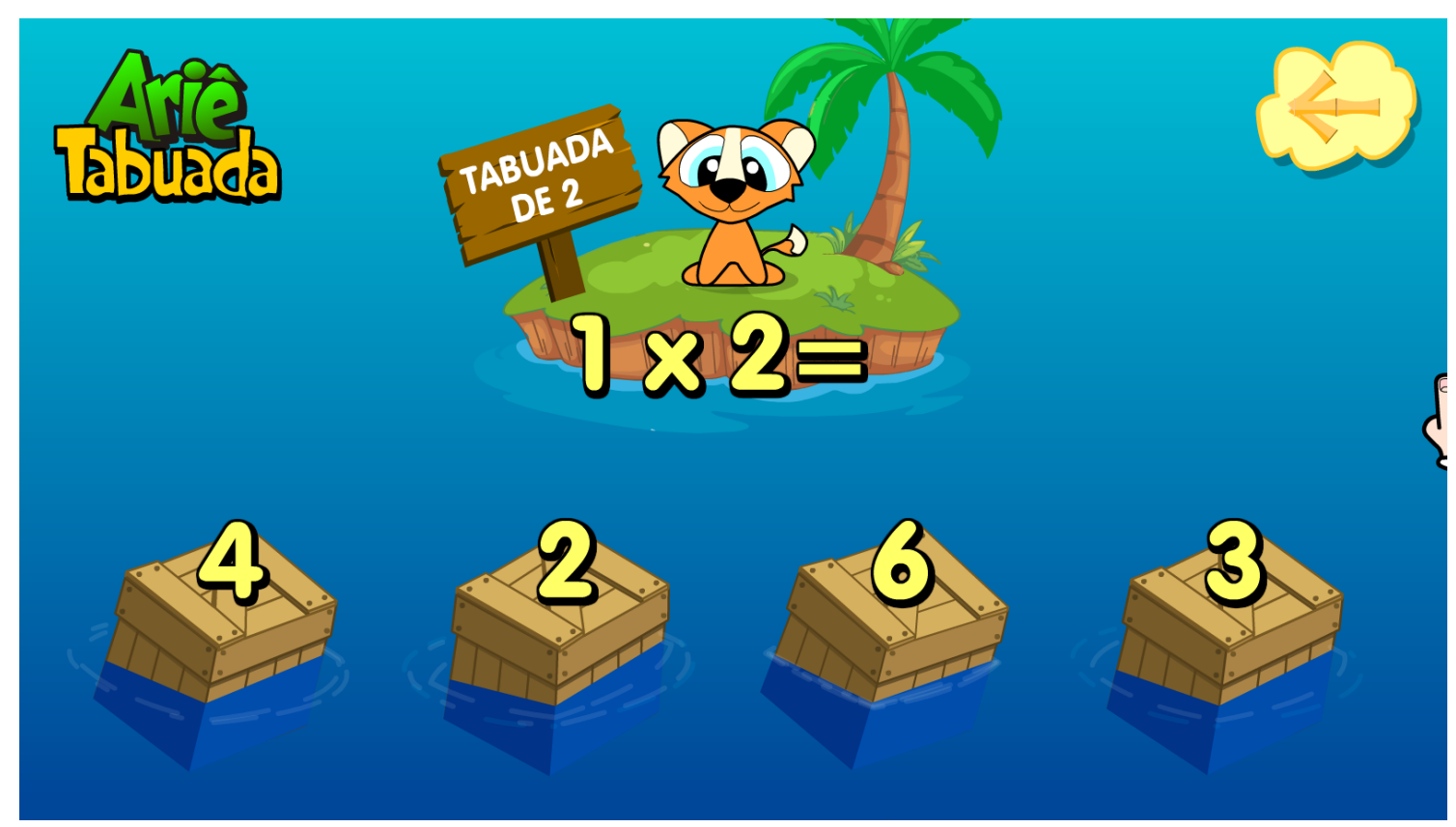

Fonte: Brincando com Ariê, 2019.

Durante o trabalho desenvolvido foi observado o desenvolvimento cognitivo dos alunos fazendo a utilização do jogo. Após o período de utilização do software "Brincando com Ariê" como ferramenta de ensino, foi aplicado novamente uma avaliação semelhante a primeira.

Os resultados entre as duas avaliações serão analisados com o auxílio do software estatístico Minitab®, para comparação dos resultados obtidos entre as avaliações 1 e 2.

A Figura 3 mostra a avaliação 1, aplicada antes do software e a avaliação 2 depois da utilização do mesmo. 
Figura 3 - Avaliação 1 e 2.
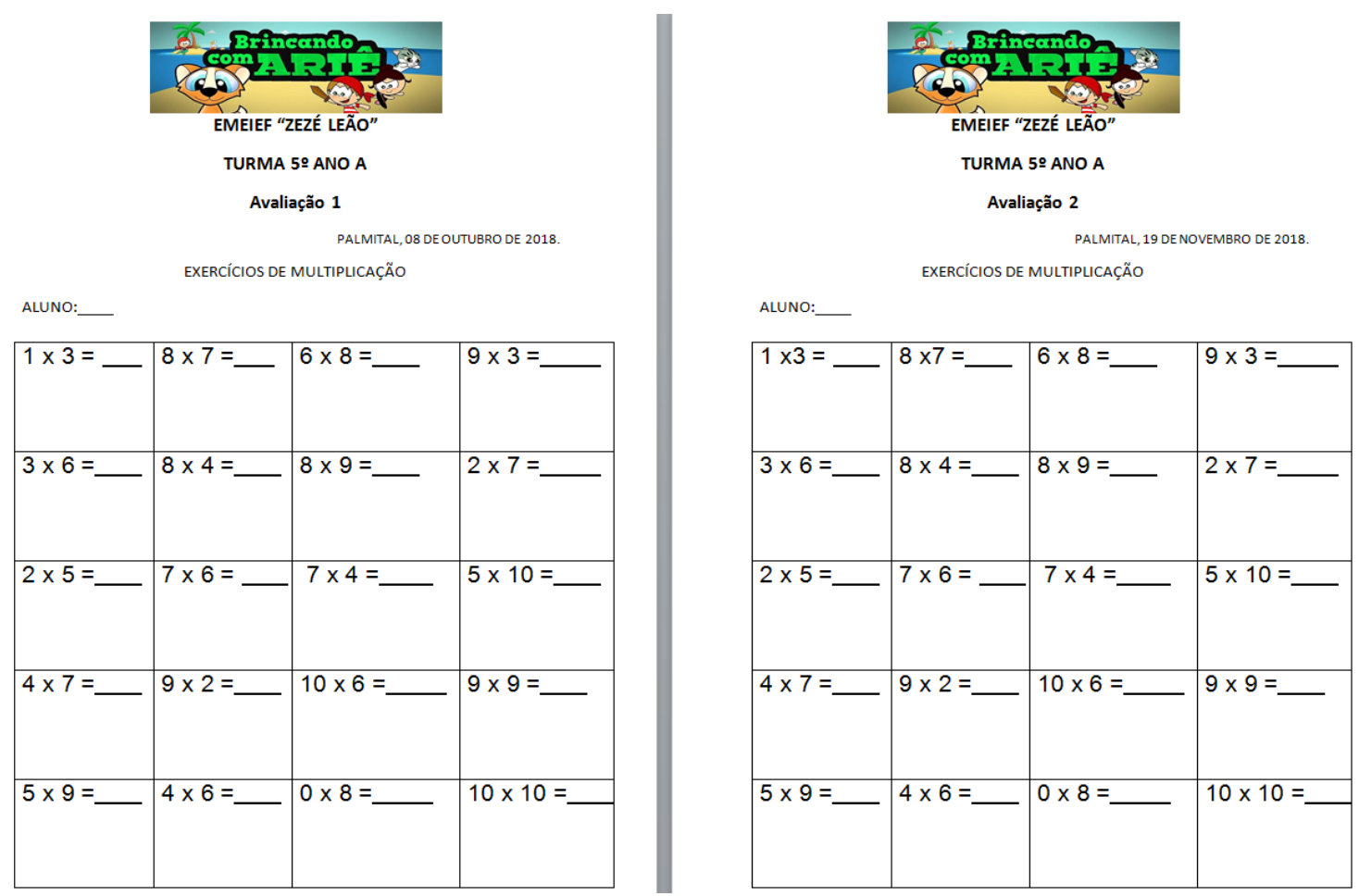

Fonte: O autor (2019).

A Tabela 1 apresenta um comparativo de acertos entre as duas avaliações, a primeira aplicada antes da aula com o uso de jogos e a segunda após a última aula com jogo.

Tabela 1 - Comparativo de acerto dos alunos nas duas avaliações.

\begin{tabular}{lll}
\hline ALUNO & ACERTOS AVALIAÇÃO 01 & ACERTOS AVALIAÇÃO 02 \\
\hline ALUNO 01 & 17 & 19 \\
\hline ALUNO 02 & 15 & 18 \\
\hline ALUNO 03 & 13 & 15 \\
\hline ALUNO 04 & 14 & 18 \\
\hline ALUNO 05 & 19 & 19 \\
\hline ALUNO 06 & 11 & 17 \\
\hline ALUNO 07 & 15 & 19 \\
\hline ALUNO 08 & 6 & 6 \\
\hline ALUNO 09 & 20 & 20 \\
\hline ALUNO 10 & 18 & 20 \\
\hline ALUNO 11 & 17 & 19 \\
\hline ALUNO 12 & 17 & 18 \\
\hline ALUNO 13 & 18 & 20 \\
\hline ALUNO 14 & 20 & 20 \\
\hline ALUNO 15 & 12 & 13 \\
\hline
\end{tabular}




\begin{tabular}{lll}
\hline ALUNO 16 & 20 & 20 \\
\hline ALUNO 17 & 16 & 20 \\
\hline ALUNO 18 & 13 & 16 \\
\hline ALUNO 19 & 20 & 20 \\
\hline ALUNO 20 & 4 & 5 \\
\hline
\end{tabular}

Fonte: $\mathrm{O}$ autor (2019).

Na primeira avaliação foi constatado que dos 20 alunos da turma apenas 6 conseguiram terminar o trabalho sem grandes dificuldades.

Após a realização do experimento e análise dos resultados pode-se perceber que houve um aumento de acertos nos cálculos de multiplicação, porém alguns alunos mantiveram sua média e outros não melhoraram. Os alunos se interessaram mais, questionaram sobre as contas e os resultados, queriam saber o porquê e onde foi o erro.

A partir dos dados obtidos da Tabela 1, foi realizada a análise estatística descritiva das avaliações 1 e 2, conforme Tabela 2.

Tabela 2 - Estatística descritiva do número de acerto dos alunos

\begin{tabular}{llllll}
\hline Variável & Média & Desvio Padrão & Mediana & Mínimo & Máximo \\
\hline Avaliação 1 & 15,25 & 4,48 & 16,50 & 4,00 & 20,00 \\
\hline Avaliação 2 & 17,10 & 4,40 & 19,00 & 5,00 & 20,00 \\
\hline
\end{tabular}

Fonte: O autor (2019)

Observando a Tabela 2, nota-se uma alteração nas médias, o desvio padrão entre as avaliações 1 e 2 se mantiveram semelhantes. Com o aumento da média ocorreu um aumento da mediana e os valores mínimos e máximos não obtiveram grandes mudanças, conforme mostra a Figura 4.

Figura 4 - Gráfico de Caixa Comparativo para Avaliação 1 e Avaliação 2

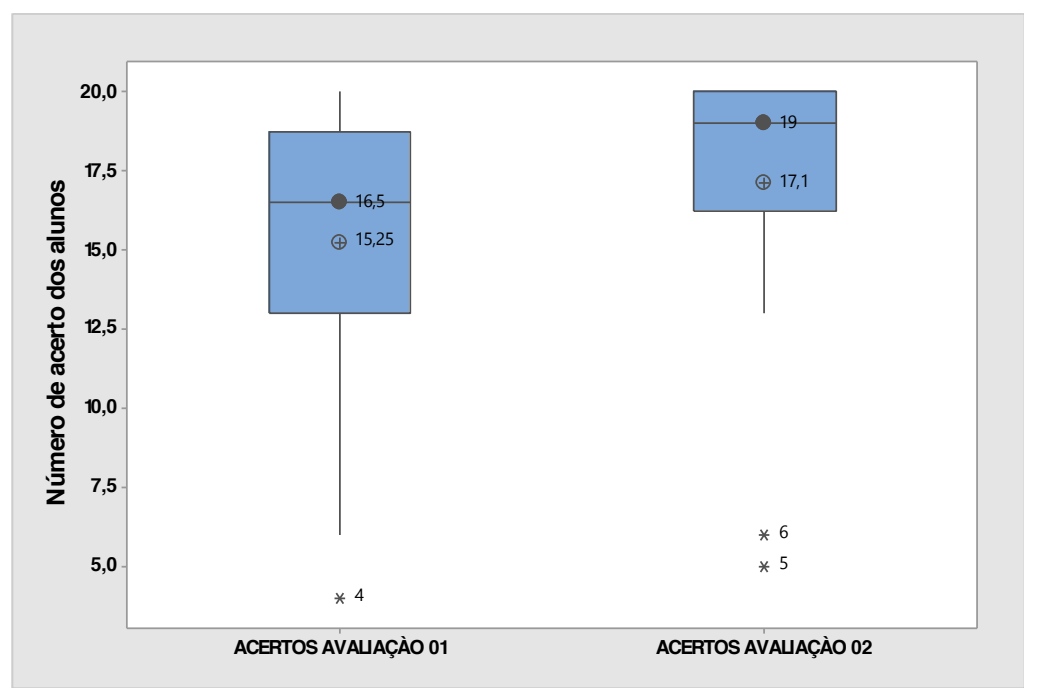

Fonte: O autor (2019). 
Nota-se na Figura 4 a existência de 2 outliers, referentes aos alunos 8 e 20 (menores notas nas avaliações). Esses alunos estão fora da normalidade dos dados, deslocando os valores de médias e aumentado o desvio padrão. Deve-se dar atenção especial para os mesmos, pois têm maiores dificuldades no aprendizado.

Para analisar o desempenho dos alunos após as duas avaliações, os alunos foram separados em níveis de aprendizagem com base na porcentagem de acerto, como observado na Tabela 3.

Tabela 3 - Desempenhos dos Alunos

\begin{tabular}{|c|c|c|c|c|}
\hline \multirow{3}{*}{ Nível } & \multirow{3}{*}{ Critério } & \multirow{3}{*}{$\begin{array}{l}\text { Porcentagem de } \\
\text { Acertos }(\%)^{*}\end{array}$} & \multicolumn{2}{|c|}{ Quantidade de Quantidade de } \\
\hline & & & Alunos - Avaliação & Alunos - Avaliação \\
\hline & & & 01 & 02 \\
\hline 1 & Excelente & $80-100$ & 11 & 16 \\
\hline 2 & Bom & $60-79$ & 6 & 2 \\
\hline 3 & regular & $40-59$ & 1 & 0 \\
\hline 4 & insuficiente & $0-39$ & 2 & 2 \\
\hline
\end{tabular}

* Porcentagem relativa ao número de afirmações corretas nas avaliações.

Fonte: $\mathrm{O}$ autor (2019).

\section{CONCLUSÕES}

Pode-se perceber que ao jogar os alunos se interessaram mais pela tabuada, houve uma competição sadia entre eles para descobrir quem iria se sair melhor nos jogos, pois ao realizarem as contas o resultado de erro ou acerto já aparecia na tela. De certa forma foi uma atividade muito diferente do ensino tradicional da sala de aula, no desenvolvimento das atividades eles acreditavam estar apenas brincando sem se dar conta do processo de aprendizagem o qual eles estavam participando. Assim, a tecnologia propiciou a eles o aprendizado de forma lúdica, sem pressão, e com interesse visível no conteúdo proposto.

Os resultados quantitativos analisados a partir da estatística comprovaram aspectos positivos da utilização do jogo educativo como ferramenta de ensino-aprendizagem, pois os resultados apresentados do trabalho realizado após o uso dos jogos foram satisfatórios. A análise comprovou que os alunos de nível 1, 2 e 3 obtiveram melhora de acerto e alguns mantiveram as médias. Os alunos de nível 4 não tiveram melhora no desenvolvimento, necessitando de acompanhamento pedagógico mais eficaz ou até mesmo outro método de ensino, pois o software não foi suficiente para melhora na aprendizagem da tabuada. 


\section{Referências}

DANI, V. L.; GUZZO, S. M. A tabuada no contexto escolar: o processo de ensino aprendizagem a partir do material manipulável e dos jogos pedagógicos. Governo do estado do Paraná, Cadernos PDE, V.1. 2013.

DOWBOR, L. Tecnologias do Conhecimento: os desafios da educação. Petrópolis, RJ: Editora Vozes Ltda., 2001.

FAGUNDES, C. C. B.; FALKEMBACH, G. M.; Jogos online na aprendizagem da tabuada. 2013. Disponível em:<

118 https://repositorio.ufsm.br/bitstream/handle/1/682/Fagundes Claudia Cristiane Bisso.pdf?sequenc

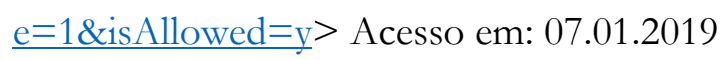

FREIRE, F. M. P.; VALENTE, J. A. Aprendendo para a vida: os computadores na sala de aula. São Paulo: Cortez, 2001.

GRÜBEL J. M.; BEZ, M.; Jogos Educativos. CINTED UFRGS.V.4, No 2, dez. 2006.

KISHIMOTO, T. M. Jogo, brinquedo, brincadeira e a educação. São Paulo: Cortez. 11 ed. 2012.

KOCH, M. Z.; As tecnologias no cotidiano escolar: uma ferramenta facilitadora no processo ensino-aprendizagem. Monografia (Especialização em Gestão Educacional) Programa de pós graduação em Gestão Educacional, Universidade Federal de Santa Maria. Sarandi, 2013.

LIMA, I. B.; PEREIRA, F. A.; Jorgildo Feijó MARTINS, J. F.; TAVARES, M. C.; J. M. F. LIBÓRIO.; BRAGA, S. R. R.; O uso dos softwares educativos no ensino aprendizagem das quatro operações matemáticas. Revista Ensino \& Pesquisa, v.13 n.01 p.109-117 jan. 2015.

OLIVEIRA, R. E. R. de. A utilização de jogos matemáticos no processo de memorizaçãa das tabuadas. Monografia (Especialização em Didática e metodologia do ensino superior), Programa de pós graduação especialização em didática emetodologia do ensino superior. Universidade do Extremo Sul Catarinense, 2008.

PEREIRA, R. A tabuada e suas implicaşões no ensino da matemática para alunos do $6^{\circ}$ ano do ensino fundamental: ficha de identificação produção didático-pedagógica - turma 2016. Governo do estado do Paraná, Disponível: < http://www.diaadiaeducação

.pr.gov.br/portals/cadernospde/pdebusca/producoes_pde/2016/2016_pdp_mat_unioeste_rosileneper eira.pdf> Acesso em: 29.01.2019.

PEREIRA, T. A.; Metodologias ativas e aprendizagem do século xxi: integração das tecnologias educacionais. Disponível em:< http://www.abed.org.br/congresso2017/trabalhos/pdf/407.pdf> Acesso em: 29.01.2019.

SANTOS, F. Z. dos; Alguns Aspectos das Interferências Pedagógicas no Ensino da Tabuada. Monografia (Especialização em Fundamentos da Educação). Curso de Especialização em Fundamentos da Educação, Universidade Federal da Paraíba. João Pessoa, 2014. 
SOUSA, V. A importância da tabuada das séries iniciais do ensino fundamental. 2018. Disponível em: <https://www.jornaldacidadeonline.com.br/noticias/8912/a-importancia-da-tabuada-nas-seriesiniciais-doensinofundamental2018> Acesso em 29.01.2019.

STAREPRAVO, A. R.; Jogando com a matemática: números e operações. Aymará. Curitiba. 2010.

VALENTE, W. R,; PINHEIRO, N V. L.; Chega de decorar a tabuada! - as cartas de Parker e a árvore do cálculo na ruptura uma tradição. Disponível em: $<$ http://sbemrs.org/revista/index.php/2011_1/article/view/157> Acesso em: 20 de março de 2019.

VESCE, G. E. P.; Ensino-aprendizagem por meio do computador. Disponível em:<

119 https://www.infoescola.com/educacao/ensino-aprendizagem-por-meio-do-computador/> 28.02.2019 VENTORINI, G.; Ensino da tabuada por meio de jogos educativos digitais. 2014. Disponível em:< https://repositorio.ufsm.br/bitstream/handle/1/11589/Ventorini Graziela.pdf? sequence=1\&isAllow $\underline{\mathrm{ed}=\mathrm{y}}>$ Acesso em 29.01.2019.

\section{Fonte}

Brincando com Ariê, 2019. Disponível em: $<\underline{\text { http://www.brincandocomarie.com.br }>\text { Acesso em }}$ ago. 2018. 